\title{
Variabilité intraspécifique chez le pin maritime (Pinus pinaster Ait) dans le sud-est de la France. II. Hétérosis et combinaison de caractères chez des hybrides interraciaux
}

\author{
A Harfouche ${ }^{1}$, P Baradat ${ }^{2}$, A Kremer ${ }^{1 *}$ \\ avec la collaboration technique de $\mathrm{J}$ Pommery ${ }^{3}$ \\ 1 INRA, laboratoire de génétique et amélioration des arbres forestiers, \\ $B P$ 45, Gazinet, 33610 Cestas; \\ 2 CIRAD, UR de biométrie, 2477, avenue du Val-de-Montferrand, BP 5035, 34032 Montpellier; \\ 3 INRA, domaine expérimental du Ruscas, 82237 Bormes-les-Mimosas, France
}

(Reçu le 25 avril 1994 ; accepté le 2 janvier 1995)

\begin{abstract}
Résumé - Cet article présente des résultats à 8,9 et 13 ans d'une expérience d'hybridation interraciale effectuée selon un schéma diallèle entre 10 provenances de pin maritime dans le sud-est de la France. L'analyse diallèle révèle une part majoritaire des effets d'aptitude spécifique à la combinaison pour des caractères de vigueur (hauteur et diamètre), de forme (coefficient de forme) et de branchaison (angle d'insertion des branches). Les effets d'aptitude générale sont, en revanche, prédominants pour l'écart à la verticalité basale et la sensibilité à Matsucoccus feytaudi. L'essentiel de la variance génétique semble se localiser au niveau hybride de provenances, l'effet famille dans hybride étant non significatif pour la plupart des caractères. Un nombre important d'hybrides manifestent un hétérosis pour la vigueur et la forme mais présentent des performances intermédiaires pour la sensibilité au ravageur. La prépondérance des effets d'aptitude spécifique et la différenciation des provenances (mise en évidence dans une étuve antérieure) ne semblent pas pouvoir expliquer totalement la fréquence élevée de cas d'hétérosis. D'autres hypothèses sont entrevues pour discuter la réponse hétérotique observée. Les hybrides Tamjout $x$ Leiria, Tamjout $x$ Cazorla et Tamjout $x$ Vivario, comparés au témoin local, combinent vigueur, forme et bonne résistance à l'insecte.
\end{abstract}

Pinus pinaster Ait / hybrides interraciaux / hétérosis / combinaison de caractères / Matsucoccus feytaudi

\footnotetext{
* Correspondance et tirés à part
} 
Summary - Intraspecific variability in maritime pine (Pinus pinaster Ait) in the south-east of France. II. Heterosis and combination of characters in interracial hybrids. This paper deals with a provenance hybridization experiment of maritime pine (Pinus pinaster Ait) at 8,9 and 13 years of age that was established in the south-east of France. The hybrids were obtained by crossing 10 provenances of the species (table l) according to a complete diallel scheme with, nevertheless, several missing combinations (fig 1). The diallel analysis showed an important proportion of specific combining ability variance for vigor characters (height and diameter), form (coefficient of dendrometric stem form) and branch habit (branch angle), whereas general combining ability effects were prevailing for the trunk straightness and sensibility to Matsucoccus feytaudi (table III). Our results revealed evidence of heterosis for most characters (table $\mathrm{V}$ ) except sensitivity to the insect pest. The important specific combining ability effects in most traits seem to be insufficient to explain the strong heterotic response observed for these characters. Some hypotheses are discussed for an understanding of such a response. A desirable parental character combination is also obtained in at least 3 hybrid combinations (Tamjout $x$ Leiria, Tamjout $x$ Cazorla, Tamjout $x$ Vivario).

\section{Pinus pinaster Ait / interracial hybrids / heterosis / desirable parental character combination / Matsucoccus feytaudi}

\section{INTRODUCTION}

Dans un article précédent (Harfouche et al, 1995), l'étude d'un échantillon de 25 provenances représentant l'ensemble de l'aire de dispersion de l'espèce a permis de mettre en évidence une importante différenciation des populations de pin maritime pour la vigueur, la forme et la sensibilité à Matsucoccus feytaudi dans le sud-est de la France. Cette différenciation est, très probablement, la traduction de différences dans les fréquences géniques et génotypiques entre populations. Cette variabilité et la complémentarité sous-jacente peuvent être mises à profit par le croisement de races suffisamment différenciées et complémentaires pour la capture d'un hétérosis et la combinaison chez les hybrides de caractères parentaux intéressants.

Les généticiens et améliorateurs forestiers se sont intéressés à l'hybridation interraciale comme méthode d'amélioration génétique des arbres forestiers à la suite des résultats obtenus sur le maïs. Parmi les premières tentatives, on citera les travaux de Johnsson sur Populus tremula $\mathrm{L}$ en Suède dès 1946 (Orr-Ewing, 1966). Depuis, les expériences se sont multipliées et ont intéressé plusieurs espèces dont le douglas au Canada (Orr-Ewing, 1966), l'épicéa commun en Suède (Nilsson, 1973) et au Canada (Morgenstern, 1973), le pin taeda aux États-Unis (Woessner, 1973), etc.

Pour la hauteur, les performances n'ont pas toujours été celles que l'on escomptait et variaient d'une espèce à l'autre et d'une station à l'autre. À quelques exceptions près (Orr-Ewing, 1966, sur le douglas ; Eriksson et llstedt, 1986, sur l'épicéa), un hétérosis (au sens de supériorité sur le meilleur parent) était rarement observé, les hybrides étant le plus souvent intermédiaires entre les 2 provenances parentes. À l'inverse, les résultats étaient très encourageants en ce qui concerne la combinaison de caractères parentaux. Par exemple, des hybrides combinant la croissance rapide de l'un des «parents» et la résistance à la rouille de l'autre «parent» furent isolés chez le pin taeda (Woessner, 1973), tandis que, chez l'épicéa, des hybrides à la fois vigoureux (caractéristique des provenances méridionales) et résistants au froid (caractéristique des provenances septentrionales) étaient obtenus (Nilsson, 1973).

Plusieurs interprétations furent proposées pour expliquer la modicité des performances hybrides en matière d'hétérosis : 
- i) L'occurrence de l'hétérosis est difficile à prédire (Knight, 1973 et Rehfeldt, 1977 in Magnussen et Yeatman, 1988), notamment lorsque des provenances à croissance lente et d'autres à croissance rapide se distribuent au hasard dans les mêmes peuplements sources de graines (Holst et Fowler, 1973).

- ii) Un croisement effectué entre des populations adaptées à des environnements différents peut engendrer un hybride qui excelle dans des milieux «hybrides» ou différents de ceux associés à ses parents (Morgenstern, 1973 ; Fowler, 1978 ; Ying, 1978 ; Hühn et Muhs, 1981 ; Magnussen et Yeatman, 1988 ; Falconer, 1989). Cette hypothèse est probablement formulée du fait de la tendance quasi générale à tester les hybrides dans les milieux de l'une ou l'autre des provenances parentes car peu de tests étaient multistationnels.

- iii) Les modèles d'analyses se basent exclusivement sur les effets additifs et de dominance des gènes. Pour des caractères en général contrôlés par un complexe de gènes, les effets d'interaction entre gènes non allèles sont probablement impliqués (Morgenstern, 1973).

De plus, des interactions génotype $x$ environnement (Hühn et Muhs, 1981 ; Eriksson et llstedt, 1986 ; Magnussen et Yeatman, 1988 ; Nilsson, 1992) viennent compliquer les analyses et limitent de ce fait le caractère général des conclusions que l'on peut en déduire.

D'une manière générale, les résultats disponibles restent fragmentaires et proviennent le plus souvent de mesures sur de jeunes plantations alors que des interactions génotype $x$ âge ne sont pas à exclure. En outre et assez fréquemment, 1 ou 2 familles hybrides supérieures au meilleur parent sont signalées mais sont trop vite occultées et les conclusions ne se réfèrent plus qu'à l'hybride moyen.

Le présent article traite d'hybrides interraciaux de pin maritime sur la base de mesures effectuées à 8,9 et 13 ans dans une plantation comparative implantée en 1982 dans le sud-est de la France (massif des Maures).

L'objectif de cette étude est double :

- détecter les combinaisons parentales aboutissant à un hétérosis. Le plan de croisement étudié comporte en effet une dizaine de populations appartenant aux grandes races géographiques du pin maritime ;

- détecter les formules hybrides permettant d'obtenir une complémentarité entre caractères. On recherchera plus particulièrement l'occurrence d'hybrides combinant une résistance appréciable à Matsucoccus feytaudi et un niveau de productivité acceptable.

\section{MATÉRIELS ET MÉTHODES}

\section{Échantillonnage de provenances et plan de croisement}

Le plan de croisement mis en ceuvre est de type diallèle complet dont les entrées sont les 10 provenances indiquées dans le tableau I.

Chaque cellule du tableau de croisements (fig 1) est constituée par la pollinisation de 5 arbres de la provenance "femelle" par un mélange de pollen de 10 arbres de la provenance "mâle». Le produit est donc un hybride moyen de provenances en référence non pas à des individus mais à des populations. Les croisements réciproques ne sont pas symétriques puisque les arbres utilisés comme mères sont complètement indépendants des individus du mélange pollinique d'une même provenance. La diagonale reconstitue les provenances pures (hybrides intraraciaux). Pour ces croisements, les 5 arbres pris comme femelles sont distincts des 10 individus du mélange pollinique (pas d'autofécondation).

Les croisements contrôlés ont été réalisés au printemps 1978 en plantation comparative de provenances sur le domaine expérimental de l'INRA de Pierroton (Gironde). Parmi les 100 hybrides de provenances attendus, 86 seulement ont été obtenus représentant au total 141 familles polycross interraciales et 13 familles polycross intraraciales. Le nombre de familles par hybride varie 
Tableau l. Situation et caractéristiques écologiques des races et provenances impliquées dans le plan de croisement.

\begin{tabular}{|c|c|c|c|c|c|c|c|}
\hline $\begin{array}{l}\text { No } \\
\text { code }\end{array}$ & $\begin{array}{l}\text { Groupe } \\
\text { goégraphique }\end{array}$ & Race & $\begin{array}{l}\text { Provenance } \\
\text { (abréviation) }\end{array}$ & $\begin{array}{l}\text { Zone géographique } \\
\text { et écologie } \\
\text { de la station }\end{array}$ & Latitude & Longitude & $\begin{array}{c}\text { Altitude } \\
(m)\end{array}$ \\
\hline 2 & Atlantique & Aquitaine & $\begin{array}{l}\text { Landes-nord } \\
\text { (LAN) }\end{array}$ & $\begin{array}{l}\text { Médoc. } \\
\text { Dune et lande }\end{array}$ & $45^{\circ} 08^{\prime} \mathrm{N}$ & $1^{\circ} 00^{\prime} \mathrm{W}$ & 40 \\
\hline 4 & Périméditerranéen & Corse & $\begin{array}{l}\text { Porto-Vecchio } \\
\text { (POV) }\end{array}$ & $\begin{array}{l}\text { Corse du Sud. } \\
\text { Sol brun superficiel, } \\
\text { roche-mère siliceuse }\end{array}$ & $41^{\circ} 39^{\prime} \mathrm{N}$ & $9^{\circ} 14^{\prime} \mathrm{E}$ & 150 \\
\hline 5 & Périméditerranéen & Corse & $\begin{array}{l}\text { Vivario } \\
\text { (VIV) }\end{array}$ & $\begin{array}{l}\text { Corse du Nord. } \\
\text { Sol brun superficiel, } \\
\text { roche-mère siliceuse }\end{array}$ & $42^{\circ} 09^{\prime} \mathrm{N}$ & $9^{\circ} 08^{\prime} \mathrm{E}$ & 600 \\
\hline 7 & Périméditerranéen & Andalousie & $\begin{array}{l}\text { Cazorla } \\
\text { (CAZ) }\end{array}$ & $\begin{array}{l}\text { Sierra andalouse. } \\
\text { Sol dolomitique } \\
\text { décarbonaté, } \\
769 \mathrm{~mm} / \mathrm{an}\end{array}$ & $37^{\circ} 32^{\prime} \mathrm{N}$ & $3^{\circ} 30^{\prime} \mathrm{W}$ & 120 \\
\hline 8 & Atlantique & Vieille Castille & $\begin{array}{l}\text { Navaleno } \\
\text { (NAV) }\end{array}$ & $\begin{array}{l}\text { Sude de la Vieille } \\
\text { Castille. } \\
424 \text { mm/an }\end{array}$ & $41^{\circ} 24^{\prime} \mathrm{N}$ & $4^{\circ} 20^{\prime} \mathrm{W}$ & 870 \\
\hline 9 & Périméditerranéen & $\begin{array}{l}\text { France } \\
\text { du sud-est }\end{array}$ & $\begin{array}{l}\text { Esterel } \\
\text { (EST) }\end{array}$ & $\begin{array}{l}\text { Massif de l'Esterel. } \\
\text { Sol brun superficiel } \\
\text { sur grès }\end{array}$ & $43^{\circ} 13^{\prime} \mathrm{N}$ & $6^{\circ} 50^{\prime} E$ & 410 \\
\hline 10 & Périméditerranéen & $\begin{array}{l}\text { (Italie } \\
\text { continentale }\end{array}$ & $\begin{array}{l}\text { Gênes } \\
\text { (GEN) }\end{array}$ & $\begin{array}{l}\text { Ligurie. } \\
\text { Sable océanique } \\
\text { sur schistes }\end{array}$ & $44^{\circ} 28^{\prime} N$ & $8^{\circ} 31^{\prime} \mathrm{E}$ & 300 \\
\hline 13 & Maghrébin & $\begin{array}{l}\text { Atlas } \\
\text { marocain }\end{array}$ & $\begin{array}{l}\text { Tamjout } \\
\text { (TAM) }\end{array}$ & $\begin{array}{l}\text { Moyen Atlas (Taza). } \\
\text { sol brun superficiel } \\
\text { sur grès caicaire, } \\
650 \mathrm{~mm} / \mathrm{an}\end{array}$ & $33^{\circ} 52^{\prime} \mathrm{N}$ & $4^{\circ} 02^{\prime} \mathrm{W}$ & 1600 \\
\hline 15 & Attantique & $\begin{array}{l}\text { Portugal } \\
\text { «type Leiria» }\end{array}$ & $\begin{array}{l}\text { Leiria } \\
\text { (LEI) }\end{array}$ & $\begin{array}{l}\text { Nord Portugal. } \\
\text { Vieille dune, } \\
764 \mathrm{~mm} / \mathrm{an}\end{array}$ & $39^{\circ} 49^{\prime} \mathrm{N}$ & $8^{\circ} 51^{\prime} \mathrm{W}$ & 50 \\
\hline 16 & Maghrébin & Tunisie & $\begin{array}{l}\text { Tabarka } \\
\text { (TAB) }\end{array}$ & $\begin{array}{l}\text { Littoral algéro-tunisien. } \\
\text { Sol brun superficiel } \\
\text { sur grès, } 1044 \mathrm{~mm} / \mathrm{an}\end{array}$ & $36^{\circ} 57^{\prime} \mathrm{N}$ & $8^{\circ} 46^{\prime} \mathrm{E}$ & 200 \\
\hline
\end{tabular}


Fig 1. Plan de croisements. Les cellules en gras désignent les combinaisons représentées dans l'expérience des Maures. Chaque croix est une famille polycross (inter ou intraraciale). Les rondes «o" désignent les croisements qui ont échoué.

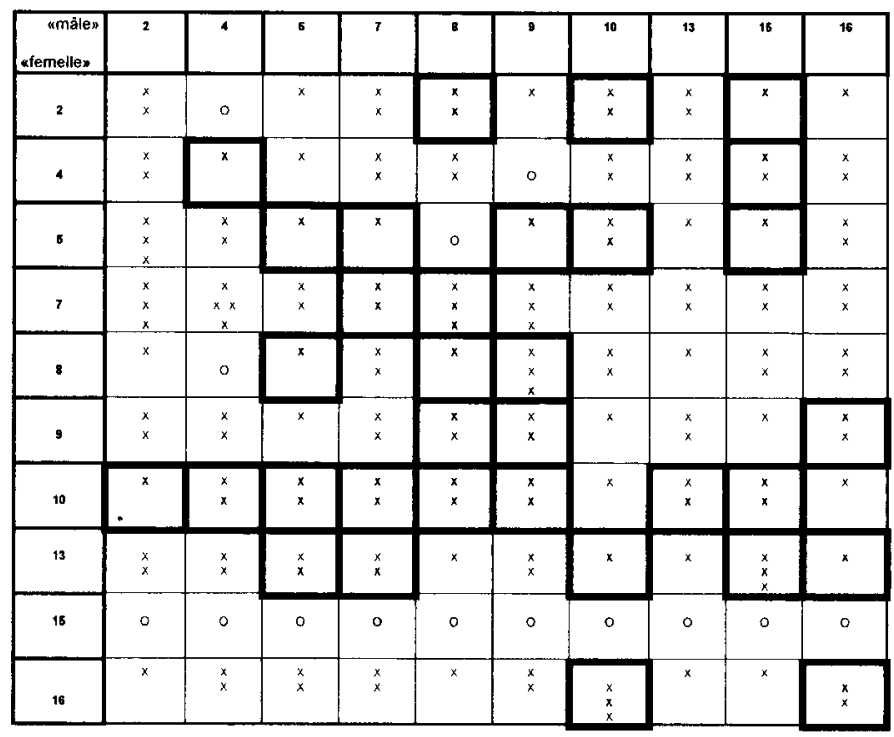

de 0 (cas des hybrides où Leiria est impliquée comme femelle notamment) à 4 (fig 1 : plan de croisements). Les familles issues de ce plan de croisements ont été réparties en une expérience bistationnelle (Landes et Maures). Seule la plantation expérimentale des Maures est traitée ici avec la remarque qu'un échantillon de $32 \mathrm{com}$ binaisons (intra et interraciales) seulement est représenté dans ce site (fig 1).

\section{Dispositif expérimental}

Le dispositif expérimental est décrit dans Harfouche et al (1995). Cependant, pour les provenances Cazorla, Leiria, Tamjout et Tabarka, des plants issus de graines récoltées in situ (pollinisation libre en forêt) ont été en plus intégrées à la plantation.

Les semis en godet ont été effectués en pépinière du Ruscas (massif des Maures) en juin 1981 et plantés en janvier 1982.

\section{Mesures et caractères observés}

Trois campagnes de mesures ont été réalisées en 1988, 1989 et 1993, c'est-à-dire aux âges de 8, 9 et 13 ans depuis le semis en pépinière. Les observations et mesures effectuées sont rapportées dans le tableau II.

\section{Traitement analytique des données}

Les caractères observés par échelle de notation de 1 à 5 (AGVI, AGVS, GRVI, GRVS, MATCOC) et EV88 (voir tableau II pour les abréviations) suivent approximativement une distribution de Poisson. Ces caractères ont été transformés par la fonction $y=\sqrt{ } x$ de façon à améliorer la normalité des distributions et stabiliser la variance résiduelle (Snedecor et Cochran, 1957 ; Dagnelie, 1973 ; Lellouch et Lazar, 1974). Dans le même but, les caractères dénombrés en pourcentage (SUR89 et SUR93) ont été transformés par la fonction $y=\arcsin \vee p / 100$.

Les données individuelles ont été ajustées à l'effet bloc selon un modèle d'analyse de variance à 2 facteurs, famille de bloc, pour prendre en compte la non orthogonalité du dispositif expérimental.

Une analyse exploratoire n'ayant pas montré de différences significatives entre les provenances récoltées in situ et les familles issues des croisements intra-races, celles-ci sont confondues 
Tableau II. Observations et mesures.

\begin{tabular}{lll} 
Campagne 1988 & Campagne 1989 & Campagne 1993 \\
\hline Hauteur totale (H88) & Hauteur totale (H89) & Hauteur totale (H93) \\
$\begin{array}{l}\text { Écart angulaire } \\
\text { à la verticalité (EV88) }\end{array}$ & $\begin{array}{l}\text { Taux de survie 1989 } \\
\text { (SUR89) }\end{array}$ & Diamètre à 1,30 m du sol (D1,30)
\end{tabular}

Taux de survie 1988

Diamètre à mi-hauteur $(\mathrm{DO}, 5)$

(SUR88)

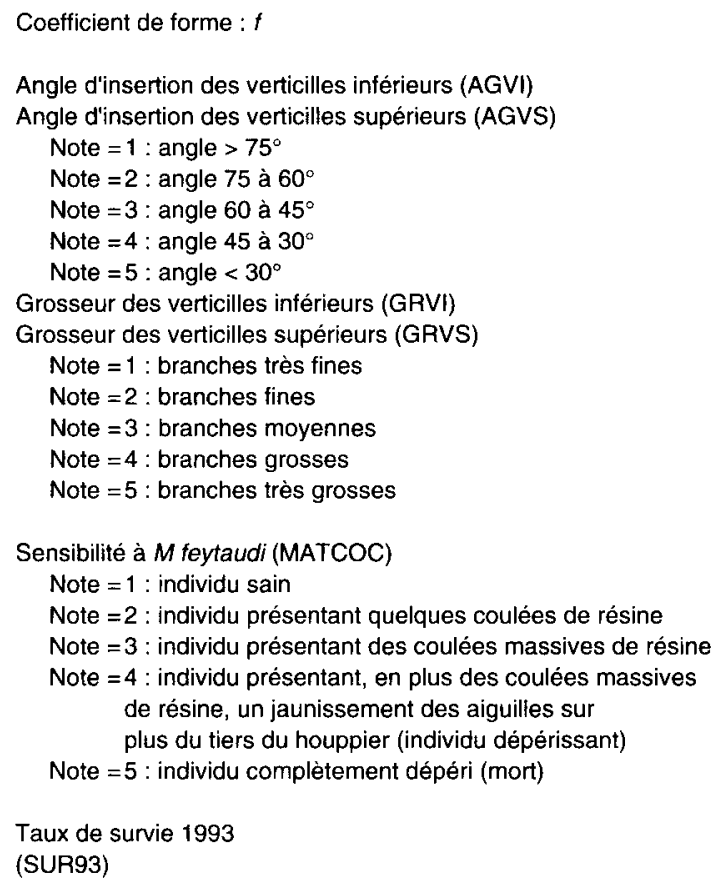

dans les analyses et sont sensées représenter la provenance considérée.

\section{Étude des aptitudes à la combinaison des provenances. Décomposition de la variabilité génétique totale}

L'analyse de la variabilité génétique a été conduite de 2 manières :

- i) analyse diallèle dans le but d'estimer les valeurs en croisement des provenances ainsi que les variances d'aptitude générale et spécifique à la combinaison ;

- ii) analyse des effets hybride interracial et famille polycross dans hybride pour estimer les performances des hybrides interraciaux et les variances de ces 2 effets.

Pour ces 2 analyses, nous avons supposé l'absence d'effets réciproques et utilisé de ce fait des modèles avec réciproques confondus. D'autre part, dans les 2 cas, les éléments de la diagonale ne sont pas pris en compte pour l'estimation des composantes de la variance. Ces «selfs" 
ont été, en revanche, intégrés dans la deuxième analyse pour l'estimation des valeurs propres des provenances.

L'analyse diallèle est effectuée selon un modèle d'analyse de variance à 2 facteurs de classification (provenance mère et provenance père à 10 niveaux) qui est une extension du modèle Henderson III adaptée de Garretsen et Keuls (Baradat, 1982). L'écriture de ce modèle est la suivante sous l'hypothèse d'absence d'effets réciproques :

$$
Y_{i j k}=\mu+g_{i}+g_{j}+s_{i j}+e_{i j k}
$$

où $Y_{i j k}$ est la performance de l'individu $k$ ajustée à l'effet bloc; $g_{i}$, l'effet d'aptitude générale à la combinaison de la provenance mère $; g_{j}$, l'effet d'aptitude générale à la combinaison de la provenance père ; $s_{i j}$, l'effet d'aptitude spécifique à la combinaison du couple $(i, j)$; et $e_{i j k}$, le résidu qui intègre l'erreur vraie, l'effet famille dans hybride et l'interaction famille $\mathrm{x}$ bloc quand elle existe.

En termes de composantes de la variance, ce modèle s'écrit :

$$
\sigma^{2} T=2 \sigma_{A G C}^{2}+\sigma_{A S C}^{2}+\sigma^{2} E
$$

dans l'hypothèse où $\sigma_{g i}^{2}=\sigma_{g i}^{2}=\sigma^{2}{ }_{A G C}$ (absence d'effets réciproques).

Dans ce modèle, $\sigma^{2} T$ est la variance totale, $\sigma^{2}{ }_{A G C}$ et $\sigma^{2}{ }_{A S C}$, les variances d'aptitude générale et spécifique à la combinaison, $\sigma^{2} E$ la variance résiduelle.

Nous nous sommes intéressés au rapport $r=$ $\left(2 \sigma^{2}{ }_{A G C} 2 \sigma^{2}{ }_{A G C}+\sigma^{2}{ }_{A S C}\right)^{\star} 100$ qui exprime l'importance de la variance des effets d'aptitude générale à la combinaison dans la variance génétique totale.

La stabilité génétique en croisement des provenances est étudiée à l'aide des interactivités individuelles relatives (Baradat, 1989). Celles-ci s'expriment comme la part de la somme des carrés des l'aptitude spécifique à la combinaison expliquée par chaque provenance de la manière suivante :

$$
\left[\frac{1}{n_{i . .}} \sum_{j=1}^{p} n_{i j .}\left(\hat{Y}_{i j .}-\hat{g}_{j}-\hat{g}_{j}-\hat{\mu}\right)^{2}\right.
$$

$$
\left.\sum_{i=1}^{p} \frac{1}{n_{i .}} \sum_{j=1} n_{i j .}\left(\hat{Y}_{i j .}-\hat{g}_{i}-\hat{g}_{j}-\hat{\mu}\right)^{2}\right]^{\star} 100
$$

où $p$ est le nombre de provenances en croisement ; $n_{i}$, l'effectif des descendants hybrides de la provenance $i$ croisée avec les $(p-1)$ autres provenances; $n_{i j}$, l'effectif des descendants communs du couple de provenances $(i, j)$. Les estimations entre parenthèses sont issues du modèle [1].

L'analyse des effets hybride interracial et famille polycross dans hybride a été réalisée selon un modèle d'analyse de variance non orthogonale à 2 facteurs hiérarchisés (hybride interracial et famille polycross dans hybride) qui s'écrit :

$$
Y_{(i j) k l}=\mu+c_{(i j)}+f(c)_{(i j) k}+e_{(i j) k l}
$$

où $y_{(i j) k l}$ est la performance de l'individu / de la famille $k$ dans l'hybride (ij); $c(i j)$, l'effet de l'hybride $(i j) ; f(c)_{(i j) k}$, l'effet de la famille $k$ dans le croisement (ii) (cet effet majore l'erreur dans le modèle diallèle) ; $e_{(i j) k l}$, le résidu associé à l'individu $l$.

Dans ce cas, la variance se décompose comme suit :

$$
\sigma^{2} T=\sigma^{2} C+\sigma^{2} F(C)+\sigma^{2} E
$$

où $\sigma^{2} c$ est la variance de l'effet hybride; $\sigma^{2} F(C)$ la variance de l'effet famille polycross dans hybride ; les autres termes ont la même signification que précédemment.

Nous avons calculé le rapport $q=\left(\sigma^{2} F(C) / \sigma^{2} C\right.$ $\left.+\sigma^{2} F(C)\right)^{*} 100$ qui exprime le pourcentage de variance de l'effet famille dans hybride dans la variance génétique totale.

Le modèle [1] d'analyse diallèle est une décomposition de l'effet hybride du modèle [2]. En effet :

$$
\begin{aligned}
c_{(i j)} & =Y_{i j .}-\bar{Y}_{\ldots .}=\left(Y_{i j .}-\bar{Y}_{i .}-\bar{Y}_{. j .}+\bar{Y}_{\ldots} . .\right) \\
& \left.+\left(\bar{Y}_{i .}-\bar{Y}_{. .}\right)+\bar{Y}_{. j .}-\bar{Y}_{\ldots} \ldots\right)=S_{i j}+g_{i}+g_{j}
\end{aligned}
$$

et, en termes de variances,

$\sigma^{2} C=2 \sigma_{A G C}^{2}+\sigma_{A S C}^{2}$

\section{Étude de l'hétérosis}

Les provenances parentes reconstituées par croisements contrôlés et/ou les unités récoltées in situ servent de témoins pour l'évaluation des performances des hybrides interraciaux et l'estimation des effets d'hétérosis. L'hétérosis est calculé sur 
la base des performances des hybrides $\left(c_{i j}\right)$ estimées par le modèle [2] ci-dessus et des valeurs propres ( $p_{i}$ et $p_{j}$ ) des provenances parentes. II est évalué par le pourcentage de mi-distance parentale réalisé par l'hybride de la façon suivante :

$h_{i j}=\left[c_{i j}-1 / 2\left(p_{i}+p_{j}\right)\right] /\left[\sup \left(p_{i}, p_{j}\right)-1 / 2\left(p_{i}+p_{j}\right)\right] \times 100$

On a, alors, un hétérosis spécial (supériorité sur le meilleur des parents) quand $h_{i j}>100 \%$.

L'hybride est, en outre, comparé aux valeurs propres de toutes les provenances en croisements y compris les provenances parentes. $\mathrm{Si}$ l'hybride est supérieur à toutes provenances, on parlera d'hétérosis général.

\section{RÉSULTATS}

II nous semble d'abord nécessaire de préciser que notre étude ne traite pas d'hybrides issus de croisements impliquant 2 individus donnés. Par hybride, nous entendons un hybride moyen de provenances ; c'est-à-dire un hybride constitué de toutes les familles polycross du croisement de 2 provenances.

Par ailleurs, à la suite d'une forte mortalité, les valeurs propres de la provenance Tabarka ne sont disponibles que pour les caractères mesurés en 1988 et 1989 ainsi que pour les taux de survie. Les pourcentages d'hétérosis enregistrés par les hybrides correspondants ne peuvent donc être calculés que pour ces caractères. Par ailleurs, la provenance Gènes n'est pas représentée dans le dispositif, sa valeur propre ne peut donc être estimée, de même que les performances des hybrides qui en sont issus.

En outre, les survies à 8 ans (SUR88) et à 9 ans (SUR589) étant pratiquement égales, seuls les résultats concernant l'année 1989 sont présentés.

Pour les analyses de variance, les hypothèses de validité sont, a priori, supposées vérifiées. Par ailleurs, on sait que seule la non-indépendance des variables est de nature à biaiser fortement les tests $F$ et que cette hypothèse est assurée par la rando- misation totale lors de l'installation du dispositif.

\section{Aptitudes à la combinaison des provenances}

Les résultats de l'analyse diallèle sont donnés dans le tableau III. Les tests F de l'aptitude générale à la combinaison (AGC) ne sont significatifs que pour l'écart à la verticalité basale à 8 ans (EV88), GRVI, MATCOC et la survie à 13 ans (SUR93) tandis que l'effet d'aptitude spécifique à la combinaison (ASC) se révèle hautement significatif pour la plupart des autres caractères. La valeur propre des provenances est un bon prédicteur des performances de leurs descendances hybrides pour le premier groupe de caractères mais non pour le second. Les pourcentages de variance d'aptitude générale à la combinaison dans la variance génétique totale sont donnés dans le même tableau. Ils mettent en évidence l'importance des effets d'aptitude spécifique à la combinaison dans la définition des performances hybrides pour des caractères comme l'angle d'insertion des branches (AGVI, AGVS), le coefficient de forme (f), le diamètre (D1.30, D0.5) ou la hauteur. Pour ce dernier caractère, on remarquera l'accroissement progressif de ce pourcentage qui passe de 44,7 à 8 ans (1988) à 62,0 à 13 ans (1993).

La figure 2 reproduit les valeurs d'aptitude générale à la combinaison des provenances pour quelques caractères importants. Pour H93, on notera la supériorité des provenances atlantiques. Pour EV88, on peut distinguer 2 groupes de provenances aux comportements opposés :

- i) TAB, CAZ et GEN présentent une forte AGC positive, donc une probabilité élevée de transmettre leur forme médiocre à leur descendance quel que soit leur partenaire ; - ii) à l'inverse, TAM et VIV enregistrent les meilleures AGC (les plus fortement néga- 
Tableau III. Analyse de variance des effets d'aptitude générale et spécifique à la combinaison des provenances objets du plan de croisement diallèle.

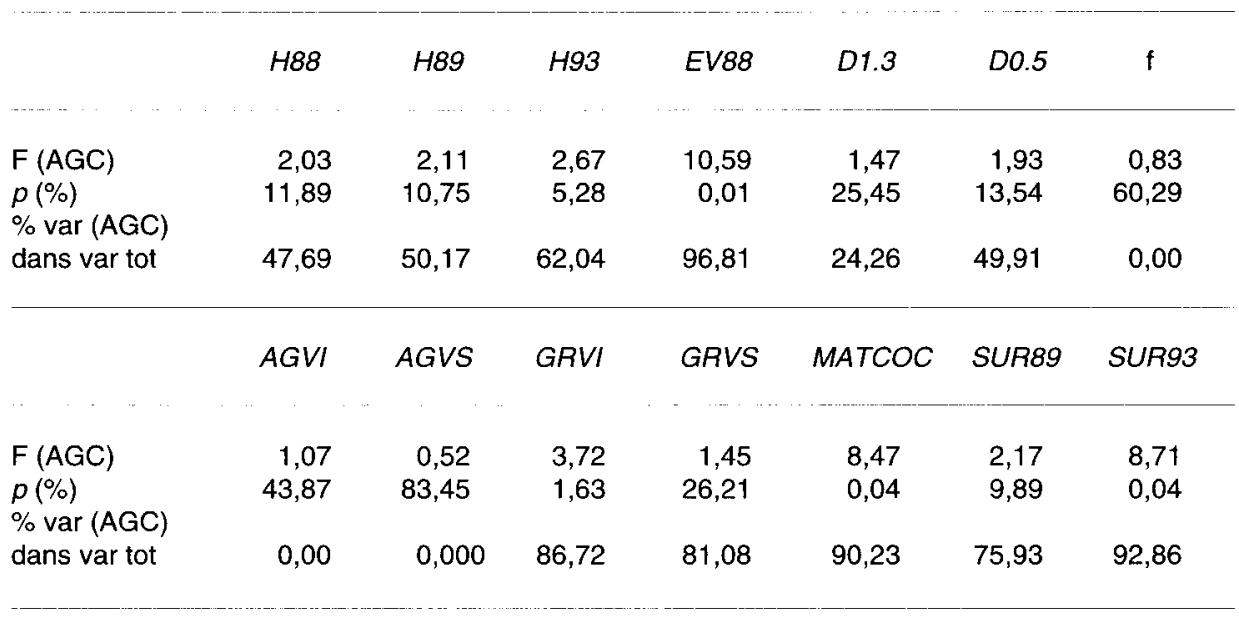

ddl AGC : 9, ddIASC : 13 ; ddl erreur : 658. Les données individuelles sont ajustées à l'effet bloc. \% var (AGC) : pourcentage de la variance totale due aux effets AGC (rapport $r$ du modèle 1 ) ; $p(\%)$ : probabilité associée au test $F$ (en pourcentage).

tives). Ces 2 provenances engendrent des hybrides moyens performants pour ce caractère.

Les autres races ont des valeurs d'AGC assez voisines de 0 et la qualité des hybrides qui en sont issus varie selon les combinaisons.

Pour la sensibilité à $M$ feytaudi, on remarque que $T A M$ et $T A B$ présentent des $A G C$ diamétralement opposées, la première montrant sa capacité à transmettre une part importante de sa résistance et la seconde conférant à ses descendants une grande sensibilité à l'insecte. Ce résultat avait été pressenti dans l'étude précédente de la variabilité intraspécifique (Harfouche et al, soumis à publication). Les valeurs des AGC des autres provenances ne sont pas significativement différentes mais on peut noter quand même la supériorité relative des races de la partie ouest de l'aire par rapport à celles de la portion est. Ce résultat confirme l'origine génétique du gradient de sensibilité ouest-est mis en évidence dans l'étude déjà citée.

On relèvera la quasi-symétrie des distributions des AGC pour SUR93 et MATCOC montrant la forte pression de sélection exercée par l'insecte aussi bien sur les provenances parentes que sur leurs hybrides.

Bien que les valeurs d'AGC ne soient pas différentes au sens statistique, on retiendra néanmoins les valeurs extrêmes pour les autres caractères:

- LAN, LEI et VIV avec de fortes AGC vs $T A B, N A V$ et $P O V$ qui enregistrent les plus faibles pour la hauteur. Ainsi, les 2 provenances corses (VIV et POV) montrent des comportements très différents, suggérant une hétérogénéité génétique de la race corse pour la vigueur.

- VIV et NAV vs TAB et CAZ pour la grosseur des branches. La qualité de la provenance Vivario pour la finesse des branches serait en grande partie transmise aux hybrides interraciaux qui en dérivent. 

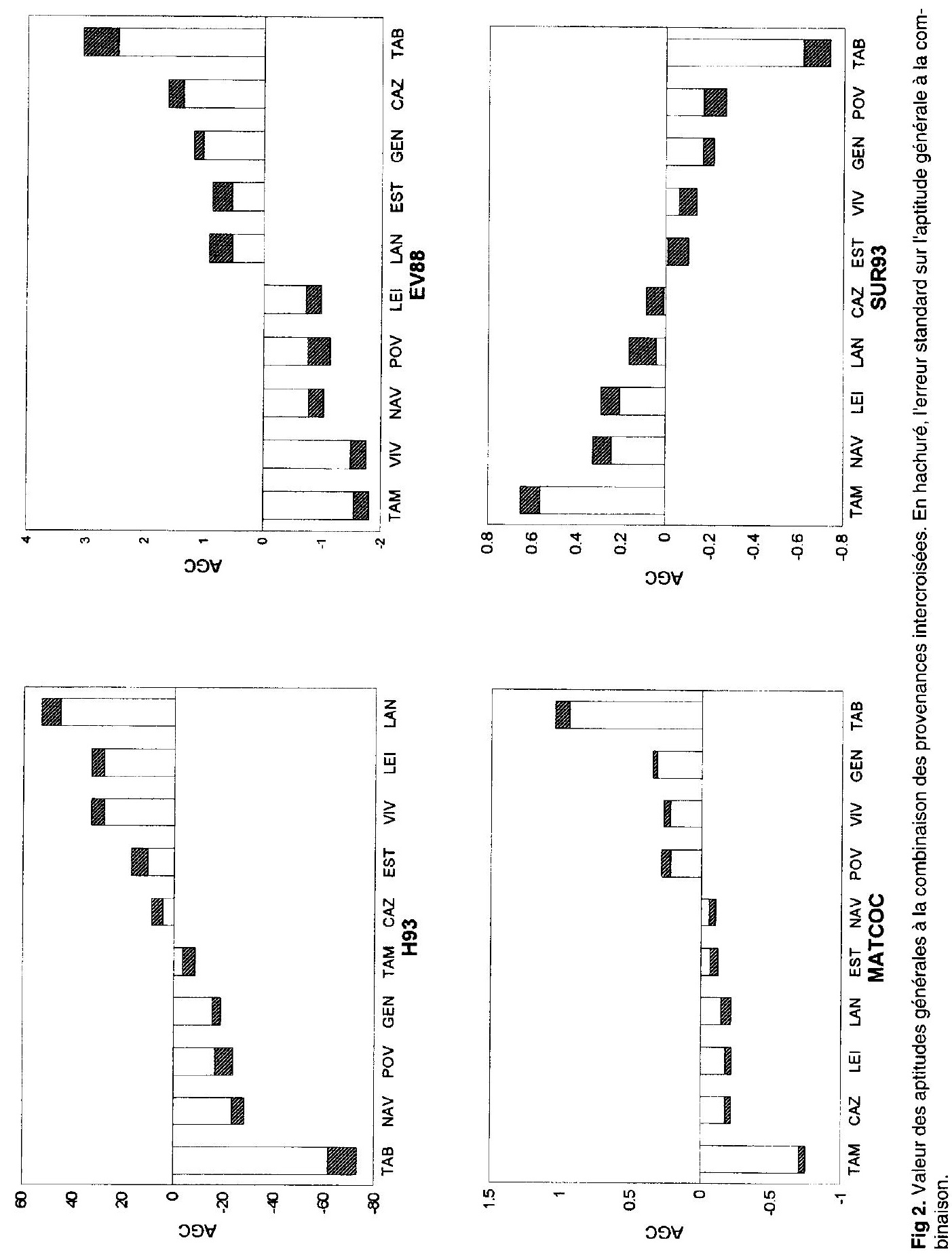
- TAB contre LEI pour l'angle d'insertion des branches et TAB contre LAN pour le coefficient de forme.

Les effets d'aptitude spécifique étant globalement significatifs pour beaucoup de caractères, il est intéressant d'examiner quelles sont les provenances qui se montrent les plus interactives (fortes ASC) et celles, au contraire, dont la valeur est relativement indépendante de la combinaison (faibles ASC). Les calculs d'interactivité individuelle relative montrent que l'on peut opposer :

- VIV $(20 \%)$ et LEI $(16 \%)$ à POV $(2 \%)$ et EST (3\%) pour la hauteur. On remarquera, pour les premières, qu'en sus d'une bonne AGC elles peuvent s'impliquer dans des combinaisons particulièrement performantes pour ce caractère.

- TAB $(20 \%)$ et VIV $(15 \%)$ à POV $(1 \%)$ et GEN $(4,5 \%)$ pour l'écart à la verticalité basale. La même remarque est à faire ici pour VIV.

- POV $(25 \%)$ et LEI (16\%) à TAM (3\%) et EST $(4,5 \%)$ pour le coefficient de forme.

- LEI $(17,5 \%)$ et VIV $(16 \%)$ à TAM $(5 \%)$ et POV $(5,5 \%)$ pour l'angle d'insertion des branches. À noter aussi la tendance, en général et en particulier pour LEI, à engendrer des hybrides aux qualités médiocres pour ce caractère de forme.

- EST $(34 \%)$ et CAZ $(14 \%)$ à POV $(1,5 \%)$ et LEI (2\%) pour la grosseur des branches.

- EST $(22 \%)$, VIV $(17,5 \%)$ et TAB $(15,5 \%)$ à $\operatorname{TAM}(2,5 \%)$, LAN $(4,5 \%)$, NAV et LEI $(6 \%)$ pour la sensibilité à $M$ feytaudi. Les dernières donneront à général les hybrides les plus résistantes (bonne stabilité).

\section{Décomposition de la variabilité génétique totale. Variabilité entre hybrides et entre familles dans hybride}

Sauf pour les angles d'insertion (AGVI et AGVS) et dans une moindre mesure, le coefficient de forme ( $f$ ) et la grosseur des branches aux verticilles inférieurs (GRVI), les valeurs du rapport $q$ sur le tableau IV montrent que la variance entre familles (à l'intérieur d'un hybride entre provenances) ne représente qu'une faible part de la variabilité génétique par rapport à la variance

Tableau IV. Analyse de variance en modèle hiérarchisé des facteurs hybrides moyens de provenance et famille polycross dans hybride.

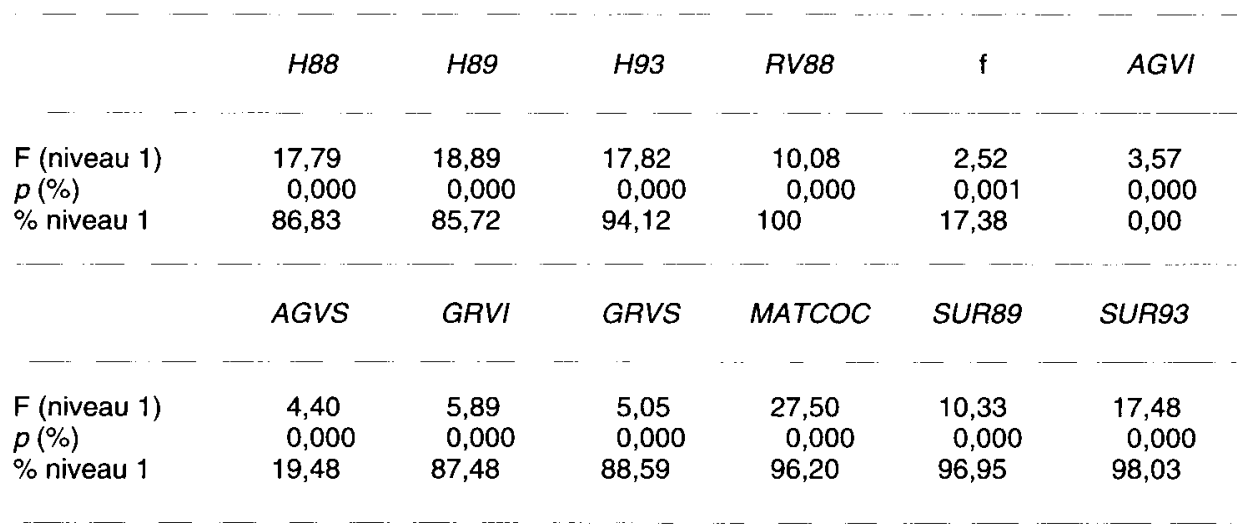

ddl niveau $1: 31$; ddl niveau $2: 14$; ddt erreur : $1049 ; \%$ niveau 1 : pourcentage de variance totale due à l'effet provenance (rapport q du modèle 2) ; $p(\%)$ : probabilité associée au test $F$ (en pourcentage). 
entre hybrides. En d'autres termes, l'effet hybride moyen de provenances est toujours nettement plus significatif que l'effet hybride spécifique (particulier à un arbre mère croisé à plusieurs père par polycross) à l'intérieur d'un hybride moyen de provenances).

\section{Hétérosis}

Le tableau $\mathrm{V}$ rapporte, pour la hauteur totale à 13 ans $(\mathrm{H} 93)$ et MATCOC, les valeurs propres des provenances, les performances des hybrides ainsi que les pourcentages de mi-distance parentale captée par l'hybride.

\section{Hauteur totale à 13 ans (H93)}

\section{Hétérosis général}

On enregistre, pour ce caractère, une fréquence assez élevée de cas d'hétérosis général. Quatre hybrides, LEI x LAN (différence significative au seuil de 5\%), CAZ x VIV, EST $x$ VIV, TAM $x$ VIV dépassent en effet la meilleure provenance (LAN) (tableau IV). Les hybrides LEI se révèlent particulièrement performants. On remarquera en outre le bon comportement de certains hybrides VIV malgré la performance modeste de la provenance.

\section{Hétérosis spécial}

Neuf hybrides sur un total de 23 montrent un hétérosis spécial pour la hauteur totale avec des valeurs de $h_{i j}$ (pourcentage de la midistance parentale captée) allant de $800 \%$ pour EST x VIV à $136 \%$ pour LEI x TAM.

\section{Écart à la verticalité basale à 8 ans (EV88)}

\section{Hétérosis général}

Les résultats montrent l'existence de 3 hybrides surpassant la meilleure provenance (TAM). Il s'agit de TAM $\times$ LEI, TAM x VIV et
NAV $x$ VIV. Les races originaires de Corse (surtout Vivario), ainsi que celles de la partie sud-ouest de l'aire (TAM et LEI en particulier) se révèlent, en général, spécialement intéressantes. Les races de la partie orientale (TAB, GEN, EST), à l'opposé, produisent les combinaisons les plus médiocres.

\section{Hétérosis spécial}

Quatre combinaisons expriment une supériorité par rapport à leur meilleur parent, LEI $x$ POV venant s'ajouter aux 3 cas d'hétérosis général déjà signalés. L'hétérosis est très net pour TAM $\times$ VIV avec une réalisation de $210 \%$ de la mi-distance parentale alors qu'il n'est que de $130 \%$ pour TAM $x$ LEI. L'hybride TAB $\times$ EST se montre particulièrement mauvais (infériorité au plus mauvais des parents).

II faut remarquer, pour ce caractère, l'existence d'un hétérosis malgré une faible variance de l'aptitude spécifique à la combinaison signalée précédemment dans l'analyse diallèle (test $F$ non significatif). Cette variance étant une moyenne, une explication semble être l'existence de variances imporrantes dans certaines provenances. En particulier, VIV et TAM, qui sont les plus impliquées dans les combinaisons hétérotiques, expliquent $25 \%$ de la variance totale de l'aptitude spécifique à la combinaison.

\section{Sensibilité à M feytaudi (MATCOC)}

On n'observe aucun effet d'hétérosis au sens favorable pour ce caractère. On enregistre, en revanche, 3 hybrides aux performances inférieures à celles du parent le plus sensible : EST x NAV, EST $\times$ VIV et LEI $x$ LAN. Pour ce dernier, dont il faut quand même relever la sensibilité moyenne $(1,96)$ inférieure à la moyenne générale $(2,06)$, I'hétérosis défavorable semble dû à une faible différence entre les valeurs propres des 2 provenances $(1,74$ et 1,75$)$. On remarquera la tendance du témoin local 
Tableau V. Valeurs propres des provenances, performances des hybrides de provenances et hétérosis réalisé.

$$
\text { Hybride }
$$

Pi

$$
P i+P j / 2
$$

Cij

A. $H 93(\mathrm{en} \mathrm{m})$
LEI $\times$ LAN
CAZ $\times$ VIV
EST $\times$ VIV
TAM $\times$ VIV
TAM $\times$ LEI
LEI $\times$ POV
NAV $\times$ LAN
LEI $\times$ GEN
GEN $\times$ LAN
GEN $\times$ CAZ
NAV $\times$ VIV
LEI $\times$ VIV
EST $\times$ NAV
GEN $\times$ NAV
GEN $\times$ VIV
TAM $\times$ CAZ
GEN $\times$ EST
TAB $\times$ GEN
GEN $\times$ POV
TAM $\times$ GEN
NAV $\times$ CAZ
TAB $\times$ TAM
TAB $\times$ EST

B. MATCOC (note de 1 à 5)

\section{TAB $\times$ GEN}

GEN $\times$ VIV

GEN $x$ POV

EST $\times$ VIV

GEN $\times$ NAV

TAB $\times$ TAM

GEN $\times$ CAZ

TAB $\times$ EST

LEI $\times$ POV

GEN $x$ LAN

LEI X VIV

EST $x$ NAV

NAV $x$ VIV

GEN $\times$ EST

LEI $\times$ GEN

LEI $x$ LAN

NAV $\times$ CAZ

CAZ X VIV

NAV $x$ LAN

TAM $\times$ GEN

TAM $\times$ VIV

TAM $\times$ CAZ

TAM $\times$ LEI

$\begin{array}{ll}5,75 & 5,85 \\ 4,95 & 5,20 \\ 5,30 & 5,35 \\ 4,65 & 5,00 \\ 4,65 & 5,20 \\ 5,75 & 5,77 \\ 5,30 & 5,50 \\ 5,75 & ? \\ ? & ? \\ ? & ? \\ 5,30 & 5,37 \\ 5,75 & 5,60 \\ 5,30 & 5,30 \\ ? & ? \\ ? & ? \\ 4,65 & 4,80 \\ ? & ? \\ ? & ? \\ ? & ? \\ 4,65 & ? \\ 5,30 & 5,10 \\ ? & ? \\ ? & ?\end{array}$

$?$

$?$

?

$?$

$?$

?

1,74

?

1,74

2,29

2,14

?

1,74

1,74

2,14

1,84

2,14

1

1

1

\section{$?$}

$?$

?

2,36

?

$?$

$?$

?

2,33

?

2,10

2,21

2,30

?

?

1,74

1,99

2,15

1,94

?

1,73

1,42

1,37
6,65

6,20

6,15

6,10

5,95

5,85

5,8

5,70

5,70

5,65

5,65

5,60

5,6

5,50

5,50

5,45

5,45

5,25

5,20

5,20

5,10

4,95

4,85

3,51

3,01

2,65

2,56

2,54

2,52

2,49

2,48

2,47

2,44

2,35

2,31

2,28

2,22

2,20

1,96

1,91

1,87

1,81

1,81

1,58

1,46

1,34
Pj

Hij $(\%)$

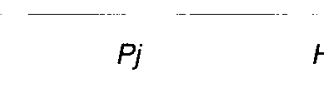

$\begin{array}{ll} & \\ 6,00 & 533 \\ 5,45 & 400 \\ 5,45 & 800 \\ 5,45 & 244 \\ 5,75 & 136 \\ 5,80 & 200 \\ 6,00 & 60 \\ ? & ? \\ 5,00 & ? \\ 4,95 & ? \\ 5,45 & 350 \\ 5,45 & 0 \\ 5,30 & \text { indéfini } \\ 5,30 & ? \\ 5,45 & ? \\ 4,95 & 433 \\ 5,30 & ? \\ ? & ? \\ 5,80 & ? \\ ? & ? \\ 4,95 & 0 \\ 4,65 & ? \\ 5,30 & ?\end{array}$

$?$

2,47

2,93

2,47

2,14

1,00

1,84

2,29

2,93

1,75

2,47

2,14

2,47

2,29

?

1,75

1,84

12,47

1,75

?

2,47

1,84

1,74
?

?$$
200
$$$$
\text { ? }
$$$$
?
$$$$
\text { ? }
$$

23

?

67

125

$-0,11$

?

?

220

$-15$

$-87$

$-65$

?

$-20$

4

$-8$

$\mathrm{Pi}$ et $\mathrm{Pj}$ sont les valeurs propres des provenances parentes; $\mathrm{Cij}$, la performance de l'hybride et Hij, le pourcentage de mi-distance parentale captée par l'hybride. Les valeurs de Hij supérieures à $100 \%$ indiquent un hétérosis favorable. Les valeurs propres et performances indisponibles sont indiquées par? 
(EST) à engendrer des combinaisons très sensibles de même que la race voisine de Ligurie (GEN) et la race tunisienne de Tabarka (TAB).

La complémentation n'est favorable qu'entre TAM d'une part et CAZ, LEI et VIV d'autre part. Les hybrides les plus résistants sont (tableau IV) :

- TAM x LEI (note $=1,34$ et $60 \%$ d'individus complètement sains) ;

- TAM $\times$ CAZ (note $=1,42$ et $72 \%$ d'individus complètement sains) ;

- TAM x VIV (note $=1,73$ et $38 \%$ dindividus complètement sains).

Il est curieux de constater le bon comportement de ce dernier hybride alors que VIV affiche $0 \%$ d'arbres sains.

Il peut paraître spéculatif d'en discuter étant donné la nature des familles, mais ces résultats ne sont pas en faveur d'un contrôle génétique simple pour le caractère parce qu'il existe réellement des degrés de sensibilité entre les individus d'une même descendance qui va souvent de la résistance totale à la sensibilité extrême. Pour approcher la question avec précision, il faudrait pratiquer des croisements biparentaux et étudier les $\mathrm{F} 1$ puis la ségrégation du caractère chez les F2.

\section{Combinaison de caractères chez l'hybride}

L'intérêt de l'hybridation interraciale est d'aboutir à un produit amélioré combinant un maximum de caractères favorables que l'on a peu de chances de rencontrer réunis chez les provenances pures. Pour le cas présent, l'objectif principal est de créer un ou plusieurs hybrides combinant une résistance, sinon totale, du moins élevée, à $M$ feytaudi à une vigueur et une forme acceptables. Les résultats obtenus montrent l'occurrence d'un petit nombre d'hybrides qui répondent à cet idéotype :
- TAM x LEI enregistre la note moyenne de sensibilité la plus faible $(1,34)$ et un taux d'individus sains appréciable $(60 \%)$ tout en réalisant un gain de $11 \%$ sur la hauteur totale et de $73 \%$ pour la rectitude du tronc relativement au témoin local (Estérel);

- TAM X CAZ combine une résistance élevée à l'insecte (note $=1,46$ et $72 \%$ d'arbres sains) à une croissance en hauteur de $3 \%$ et une rectitude du tronc de près de $46 \%$ supérieures au témoin local ;

- TAM x VIV peut également paraître intéressant avec sa note de 1,73 et ses $38 \%$ d'individus sains alliés à des gains de l'ordre de plus de $14 \%$ pour la hauteur totale et de $71 \%$ pour la rectitude du tronc.

Par ailleurs, la provenance landaise montrant une bonne performance pour la résistance à Matsucoccus feytaudi (note $=1,75$ et $24 \%$ d'individus sains ; 1,83 et $25 \%$ pour Cazorla, 1,74 et $36 \%$ pour Leiria à titre de comparaison), il est à regretter que l'hybride TAM $x$ LAM n'ait pas été représenté dans l'expérience.

\section{DISCUSSION}

Il convient d'abord de discuter de la portée des résultats obtenus dans cette étude et de l'interprétation qui peut en être faite, et ce, en relation avec la nature de l'échantillon de provenances croisées, d'une part, et de la spécificité du plan de croisements, d'autre part.

Les provenances objets du plan de croisements ne constituent pas un tirage aléatoire simple des populations naturelles de pin maritime. Elles ont été choisies notamment selon le critère d'éloignement géographique. La conséquence en est que les estimations du modèle d'analyse n'ont pas de valeur inférentielle ; elles ne sont strictrement valides que pour l'échantillon de provenances en question et dans les limites du site d'expérience. 
D'autre part, la définition des composantes de la variance génétique (additive et de dominance) à partir des aptitudes à la combinaison des provenances ne peut être établie avec précision qu'au prix d'hypothèses restrictives (sous-populations provenant sans sélection de la même population panmictique de référence et ayant des taux de consanguinité comparables). Aucune de ces hypothèses ne peut être envisagée dans notre cas. Nous ne pouvons, par conséquent, nous placer au niveau du gène dans l'interprétation des aptitudes à la combinaison dans le cadre de cette étude (ce serait définir l'effet d'un gène à partir de l'effet d'une provenance dont la structure génétique reste indéfinie). Nous supposerons tout au plus une relation d'analogie statistique entre AGC et effets additifs et entre ASC et effet non additifs. De plus, comme il s'agit d'aptitudes à la combinaison de populations et non d'individus, leurs estimations ne sauraient être extrapolées à l'échelle individuelle, que ce soit pour des croisements intraprovenances ou pour des croisements interprovenances.

Pour les mêmes raisons, les valeurs d'hétérosis spécifique ne peuvent être traduites en termes de type d'action des gènes. En outre, l'hétérosis observé chez les hybrides de provenances semble un phénomène complexe qui pourrait résulter de plusieurs mécanismes génétiques et non génétiques comme il sera discuté plus loin.

Cela étant, les résultats obtenus montrent que l'essentiel de la variance génotypique se situe au niveau hybride moyen de provenances pour la plupart des caractères. Les faibles pourcentages de variance génétique expliquée par le niveau famille dans hybride illustrent clairement cela (tableau III).

Nous en retiendrons 2 conséquences principales avec la réserve qu'impose le faible nombre de degrés de liberté du niveau famille (12 ddl) :
- premièrement, le concept d'hybride moyen de provenances semble justifié pour la plupart des caractères étudiés ;

- deuxièmement, la sélection de familles polycross au sein des hybrides de provenances ne serait pas efficace pour ces mêmes caractères.

L'inverse est en revanche valable pour le coefficient de forme et l'angle d'insertion des branches pour lesquels existe une variabilité entre familles polycross très importante.

II semble s'agir, pour ce dernier, d'un caractère à forte variance individuelle (dans les provenances) pour d'autres conifères comme Pinus banksiana (Magnussen et Yeatman, 1988) ou Pinus nigra (Charon, communication personnelle). L'étude de la variabilité géographique dans le sud-est de la France nous a d'ailleurs permis de conclure de façon similaire pour le pin maritime (Harfouche et al, soumis pour publication).

L'analyse diallèle met en évidence l'importance de la variance d'aptitude spécifique dans la variance entre hybrides de provenances pour des caractères comme la hauteur (excepté en 1993), le diamètre, l'angle d'insertion des branches et le coefficient de forme. Deux raisons nous semblent expliquer ces résultats. La première est écologique : la sévérité des facteurs environnementaux dans la région d'expérience peut être considérée comme limitante pour le pin maritime et c'est dans ce type de conditions que les effets non additifs s'exprimeraient le plus (Ekberg et al, 1982 ; Falconer, 1989). Mais cela ne semble pas être le cas pour d'autres caractères (grosseurs des branches). La seconde pourrait être en relation avec la juvénilité du matériel végétal. Sous réserve que cette tendance se maintienne, on constate, en effet, une "extinction" progressive de ces effets avec l'âge. Au niveau interspécifique, des résultats similaires sont observés pour le mélèze (Pâques, 1992) où le rapport 
VAND diminue progressivement depuis la pépinière jusqu'à devenir négligeable à 6 ans. Ce phénomène traduirait l'existence d'ceffets maternels" aux premiers stades de la vie des plants (Falconer, 1989 ; Pâques, 1992). Cependant, une tendance inverse est trouvée chez l'épicéa de Sitka (Samuel, 1991, cité dans Pâques, 1992).

L'impact de ces résultats est important pour le choix de la stratégie de sélection et la mise en place de populations de production de matériel amélioré. Faudra-t-il exploiter les effets d'aptitude générale en privilégiant la création de variétés "synthétiques" ou bien ceux de l'aptitude spécifique par la sélection de combinaisons hautement performantes pour l'obtention de variétés «hybrides»?

La différenciation génétique entre provenances mise en évidence dans une étude antérieure (Harfouche et al, soumis pour publication) pour la plupart des caractères, ajoutée à l'importance des effets d'aptitude spécifique, fait du pin maritime un candidat idéal pour une hybridation interraciale. En effet, ces 2 éléments détermineraient la probabilité d'occurrence d'une réponse hétérotique (Owino et Zobel, 1977 ; Ying, 1978 ; Hühn et Muhs, 1981 ; Ekberg et al, 1982 ; Magnussen et Yeatman, 1988 ; Falconer, 1989 ; Bolstad et al, 1991).

Cette réponse semble cependant plus complexe puisque des croisements entre provenances trop divergentes ont au contraire conduit à une réduction de vigueur chez leurs hybrides, probablement due à un déséquilibre de la balance génétique chez l'hybride (Ying, 1978 ; Magnussen et Yeatman, 1988 ; Falconer, 1989). Cette balance génétique est liée à l'adaptation qui tend à fixer des groupes de gènes différents chez des populations soumises à des conditions naturelles très contrastées. Au cours de l'évolution, il s'établit un équilibre des interrelations entre loci spécifiques à chaque population et le croisement de telles populations génétiquement très différenciées peut conduire à une réduction de vigueur des hybrides $\mathrm{F} 1$ à cause de la rupture de cet équilibre dans les génomes hybrides (Falconer, 1989). Par ailleurs, l'existence de relations d'épistasie peut expliquer une partie de la réponse hétérotique observée (Morgenstern, 1973 ; Falconer, 1989). Comme c'est le cas pour la hauteur et la rectitude du fût chez des hybrides interspécifiques de mélèze (Pâques, 1992), l'aptitude générale à la combinaison des provenances parentes pourrait aussi déterminer cette réponse, celles dont cette aptitude est élevée donnant généralement les meilleurs hybrides.

\section{Hétérosis et vigueur}

Pour la hauteur totale, l'hétérosis enregistré est très net. Le même phénomène a été observé, à l'état juvénile, pour ce caractère sur des hybrides interraciaux plantés dans les Landes de Gascogne (Desprez-Loustau et Baradat, 1991). Chez d'autres espèces de conifères, les performances hybrides sont le plus souvent intermédiaires avec absence de supériorité sur le meilleur parent, comme c'est le cas chez le pin Taeda (Woessner, 1973 ; Owino et Zobel, 1977), le pin rouge (Holst et Fowler, 1973), le pin de Banks (Magnussen et Yeatman, 1988 ; Bolstad et al, 1991), le pin sylvestre (Nilsson, 1992), le pin noir (Gerber, 1989), l'épicéa commun (Nilsson, 1973 ; Hühn et Muhs, 1981 ; Ekberg et al, 1982 ; Kaya et Lindgren, 1992), l'épinette noire (Morgenstern, 1973).

Cependant, il existe, très probablement, des effets d'interaction hétérosis $x$ environnement puisque, en d'autres occasions, une vigueur supérieure des hybrides sur les provenances parentes a été constatée pour certaines de ces mêmes espèces (Nilsson, 1973, chez le pin sylvestre ; Ying, 1978 ; Eriksson et listedt, 1986, chez l'épicéa). Une réponse, à ce sujet, pourra être fournie pour le pin maritime à l'issue de mesures faites en 1993 sur la plantation d'hybrides interra- 
ciaux de Saint-Alban dans les Landes (Aquitaine).

Un hétérosis pour la hauteur a également été signalé chez le sapin de Douglas (Orr-Ewing, 1966).

\section{Hétérosis et forme}

Pour la rectitude du tronc, l'hétérosis constaté serait surtout lié à la différenciation des provenances, les effets d'aptitude spécifiques à la combinaison étant mineurs pour ce caractère.

Chez $P$ taeda (Magnussen et Yeatman, 1988), les résultats sont variables suivant les stations mais il n'existe de différences entre les hybrides et les provenances témoins que dans 2 cas sur 6 . La proportion d'arbres bien conformés était, dans un cas, plus grande et, dans un autre cas, plus faible chez les hybrides. Une forte interaction hétérosis $x$ environnement est également observée.

\section{Hétérosis et sensibilité à Matsucoccus}

Concernant la sensibilité à Matsucoccus feytaudi, les hybrides se montrent, le plus souvent intermédiaires mais il existe des cas d'hétérosis défavorables dans des croisements où la provenance Estérel est impliquée. Pour ce caractère, les effets d'aptitude générale à la combinaison prédominent mais la complémentation est favorable pour certaines combinaisons (TAM $\times$ CAZ et TAM $x$ LEI). Pour ces combinaisons, l'effet de synergie est peut être lié à une fréquence élevée de gènes favorables chez les provenances parentes.

\section{Combinaisons de caractères}

C'est dans son aspect de complémentation des caractères parentaux que l'hybridation interraciale chez les arbres forestiers a enregistré les résultats les plus prometteurs. La présente étude montre l'existence d'hybrides combinant favorablement les caractères de vigueur et de forme à la résistance à $M$ feytaudi. De nombreux cas de combinaison de caractères sont signalés chez d'autres espèces résineuses. On citera par exemple des hybrides combinant croissance rapide et résistance à la rouille chez $P$ taeda (Woessner, 1973), vigueur et résistance à la sécheresse chez la même espèce (Owino et Zobel, 1977), vigueur et résistance au froid chez l'épicéa commun (Nilsson, 1973), croissance rapide et résistance au froid chez le pin de Banks (Bolstad et al, 1991).

\section{CONCLUSION}

Cette expérience constitue une première étape qui devrait déboucher sur la sélection d'un petit nombre de provenances ou de combinaisons de provenances performantes à impliquer dans un programme de sélection récurrente réciproque en vue d'y choisir des individus supérieurs pour l'obtention de familles hybrides polycross ou bi-parentales. II faudra cependant disposer de données à un âge plus avancé de la plantation et multiplier les essais dans l'espace pour tirer des conclusions définitives.

Vis-à-vis à de $M$ feytaudi, il s'agira de progresser dans la connaissance du déterminisme héréditaire de la résistance et dans la recherche de génotypes hybrides résistants à l'insecte. Pour cela, certaines actions sont à mettre en œuvre, telles :

- l'inventaire, le clonage et le testage des individus présentant une résistance in situ dans le massif des Maures ;

- des plans de croisement individuels où Tamjout serait prise comme testeur, son génotype étant supposé connu. Cette provenance semble en effet avoir fixé les allèles de résistance à Matsucoccus feytaudi (Harfouche et al, soumis pour publication); 
- la création d'hybrides interraciaux F2 dans et entre les meilleurs hybrides F1 par le choix d'individus complètement sains.

\section{REMERCIEMENTS}

Nous exprimons nos remerciements à B Fady, directeur du domaine expérimental du Ruscas et à toute son équipe technique pour leur formidable accueil. Mention spéciale à l'équipe technique pour son application dans les mesures.

\section{RÉFÉRENCES}

Baradat P (1982) La génétique quantitative : modèles statistiques et génétiques de base. INRA, recherches forestières, laboratoire amélioration, Cestas, France

Baradat $P$ (1989) Amélioration génétique des arbres forestiers. Eléments méthodologiques. INRA, recherches forestières, laboratoire amélioration, Cestas, France

Baradat P, Pastuszka P, Durel CE (1990) Les hybrides entre races géographiques de pin maritime. For Gasc 364,10

Bolstad SB, Kang H, Guries RP, Marty TL (1991) Performance of inter- and intraprovenance crosses of jack pine in central Wisconsin. Silvae Genet 40, 124-129

Dagnelie P (1973) Théorie et méthodes statistiques. Les Presses agronomiques de Gembloux, Gembloux

Desprez-Loustau ML, Baradat P (1991) Variabilité de la sensibilité à la rouille courbeuse chez le pin maritime. Ann Sci For 48, 497-511

Ekberg I, Eriksson G, Hadders G (1982) Growth of intraand interprovenance families of Picea abies (L) Karst. Silvae Genet 31, 160-167

Eriksson G, Ilstedt B (1986) Stem volume of intra- and interprovenance families of Picea abies (L) Karst. Scand J For Res 1, 141-152

Falconer DS (1989) Introduction to quantitative genetics. Longman Scientific and Technical, Essex, UK

Gerber S (1989) Chimiotaxinomie et hybridation interraciale chez les pins noirs. Diplôme d'ingénieur agronome, INA Paris-Grignon

Harfouche A, Baradat P, Durel CE (1995) Variabilité intraspécifique chez le pin maritime (Pinus pinaster Ait). 1. Variabilité des populations autochtones et des populations de l'ensemble de l'aire de l'espèce. Ann Sci For 52, 307-328

Holst MJ, Fowler DP (1973) Selfing and provenance hybridization in red pine. Proc 14th Meet Can Tree Improv Assoc, part 2, 39-50

Hühn M, Muhs HJ (1981) Height growth of some interracial hybrids of Norway spruce using selected trees of a north and a central european provenance with special regard to between-plot-competition. Silvae Genet 30, 25-29

Ilstedt B, Eriksson G (1986) Quality of intra- and interprovenance families of Picea abies (L) Karst. Scand $J$ For Res 1, 153-166

Kaya Z, Lindgren $D$ (1992) The genetic variation of interprovenance hybrids of Picea abies and possible breeding consequences. Scand J For Res 7, 15-26

Lellouch J, Lazar P (1974) Méthodes statistiques en expérimentation biologique. Flammarion, Médecine Science, Paris

Magnussen S, Yeatman CW (1988) Provenance hybrids in jack pine: 15-year results in eastern Canada. Sivae Genet 37, 206-218

Morgenstern EK (1973) Eariy results of provenance hybridization in black spruce. Proc 14th Meet Can Tree Improv Assoc, part 2, 27-37

Nilsson B (1973) Recent results of interprovenance crosses in Sweden and the implications of breeding. Proc 14th Meet Can Tree Improv Assoc, part 2, 3, 1426

Nilsson JE (1992) Growth and survival of spontaneous provenance hybrids of Pinus sylvestris. Scand J For Res 7, 193-203

Orr-Ewing AL (1966) Inter- and intraspecific crossing in Douglas fir, Pseudotsuga menziesii (Mirb) Franco. Silvae Genet 15, 121-126

Owino F, Zobel B (1977) Genotype x environment interaction and genotypic stability in loblolly pine. Silvae Genet 26, 114-116

Pâques $L$ (1992) Results and future trends in larch breeding on the basis of provenance research. Proc Cent Meet IUFRO Work Part S2 02-07, Berlin, 1992

Pearce SC (1965) Introduction à la statistique en biologie. INRA, Paris (traduction de JP Doazan, 1978)

Snedecor GW, Cochran WG (1957) Méthodes statistiques. Association de coordination technique agricole (traduction de $\mathrm{H}$ Boelle et $\mathrm{E}$ Camhaji)

Woessner RA (1973) Interprovenance crosses of loblolly pine. Proc 14th Meet Can Tree Improv Assoc, part 2, 12-25

Ying CC (1978) Height growth of interprovenance crosses in white spruce, Picea glauca (Moench) Voss. Sivae Genet 27, 226-229 